\title{
Pd(0)-Catalyzed cross-coupling reactions of 2-indolylzinc halides. A convenient route to indolo[2,3-a]quinolizidines
}

\author{
Mercedes Amat,* Núria Llor, Grigorii Pshenichnyi, and Joan Bosch \\ Laboratory of Organic Chemistry, Faculty of Pharmacy, University of Barcelona, \\ Barcelona 08028, Spain \\ E-mail: amat@farmacia.far.ub.es
}

This work is dedicated to Prof. Marcial Moreno-Mañas on the occasion of his $60^{\text {th }}$ birthday (received 15 Dec 01; accepte 14d Feb 02; published on the web 22 Feb 02)

\begin{abstract}
The generation of 4-, 5-, and 6-methoxy-3-indolylzinc chlorides 3a-c and their Pd(0)-catalyzed cross-coupling reactions with 2-halopyridines to give 4-, 5-, and 6-methoxy substituted 2-(2pyridyl)indoles $\mathbf{4 a - c}$ is reported. 2-(2-Pyridyl)indole $\mathbf{8}$ is converted to indolo[2,3-a]quinolizidine 15. The key step is the regioselective cyclization on the indole 3-position of a thionium ion generated by Pummerer reaction from an appropriately substituted 2-(2-piperidyl)indole.
\end{abstract}

Keywords: Indoloquinolizidine, palladium(0), cross-coupling, Zinc, bromopyridine, Pummererreaction

\section{Introduction}

The indolo[2,3-a]quinolizidine ring system is often embedded in the structure of many naturally occurring compounds exhibiting a wide range of biological activities. ${ }^{1}$ Although at the present time there is a plethora of synthetic strategies for the elaboration of indoloquinolizidine derivatives, most approaches have relied on the generation of the $\mathrm{C}_{12 \mathrm{a}}-\mathrm{C}_{12 \mathrm{~b}}$ bond from appropriate $\mathrm{N}$-[2-(3-indolyl)ethyl]piperidines or the elaboration of the piperidine $\mathrm{D}$ ring in the last steps of the synthesis. ${ }^{2}$

In previous works ${ }^{3}$ we have reported the preparation of 2-(2-pyridyl)indoles by $\mathrm{Pd}(0)$-catalyzed cross-coupling $^{4}$ of 1-(benzenesulfonyl)-2-indolylzinc chloride and a variety of 2-chloro- and 2bromopyridines bearing substituents of different electronic nature, and we have explored a procedure for their conversion into indoloquinolizidine derivatives. ${ }^{3 a, 5}$ This approach involves the formation of the $\mathrm{C}_{7}-\mathrm{C}_{7 \mathrm{a}}$ bond by intramolecular alkylation of the indole 3-position from a 2(2-piperidyl)indole as the key step, a transformation for which no efficient methods had been described at the beginning of our studies. In this paper we report the generation of 4-, 5-, and 6- 
methoxy substituted 2-indolylzinc chlorides ${ }^{6}$ and their use in $\operatorname{Pd}(0)$-catalyzed cross-coupling reactions leading to 2-(2-pyridyl)indoles bearing methoxy groups on the indole ring, which are of interest in the synthesis of hydroxy or methoxy substituted indoloquinolizidine alkaloids such as those depicted in Figure 1. Additionally, we report an efficient conversion of 2-(2pyridyl)indole 9 to methyl indolo[2,3-a]quinolizidine-2-carboxylate 15, thus establishing the usefulness of our method for preparing indoloquinolizidines.
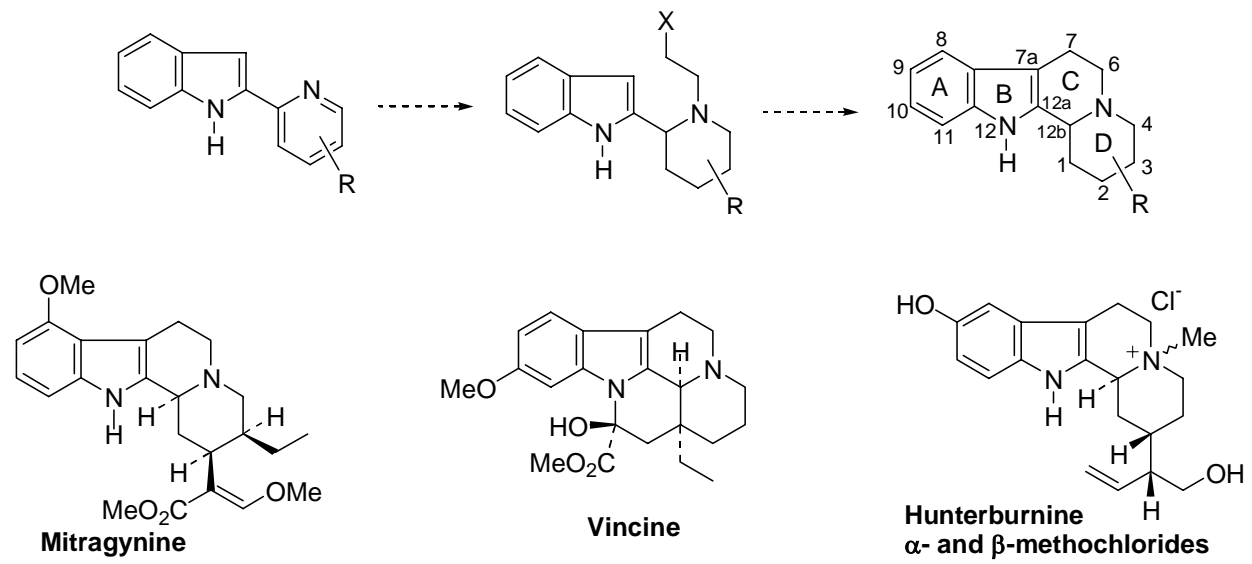

Figure 1

\section{Results and Discussion}

The required 4-, 5-, and 6-methoxyindoles 1a-c were prepared by the procedure of Batcho and Leimgruber, $^{7}$ from the corresponding methylnitroanisole derivatives. A subsequent sulfonylation was carried out under phase-transfer conditions ${ }^{8}$ to give the protected methoxyindoles 2a-c in excellent yields. Regioselective litiation of 2a-c with LDA, followed by transmetalation with anhydrous $\mathrm{ZnCl}_{2}$ afforded 2-indolylzinc chlorides 3a-c, which were allowed to react with 2bromo-4-methylpyridine in the presence of $\operatorname{Pd}(0)$ in refluxing THF. The best conditions were found to be the use of 0.1 equivalent of $\mathrm{Pd}\left(\mathrm{PPh}_{3}\right)_{4}$ with respect to the 2-halopyridine. In this manner, methoxy-2-(2-pyridyl)indoles 4a-c were isolated in 64\%, 65\%, and 80\% yield, respectively. Finally, the deprotected methoxy-2-(2-pyridyl)indoles 5a-c were obtained in good yields by treatment of 4a-c under alkaline conditions. The new methoxy substituted 2indolylzinc halides 3a-c reported here can be useful intermediates for the synthesis of indole derivatives. 


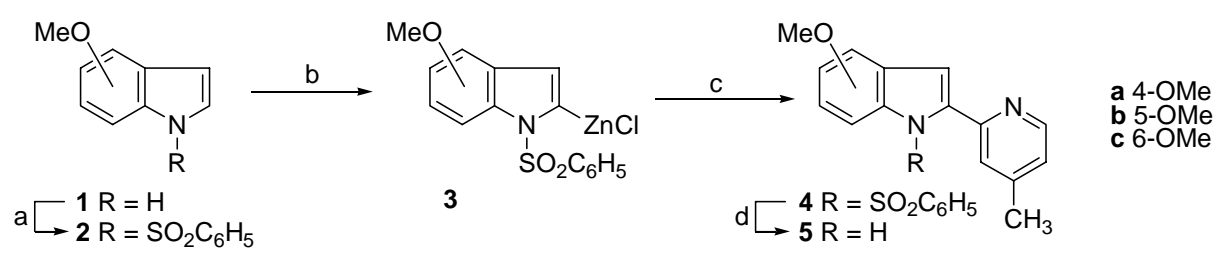

Reagents and conditions: (a) $\mathrm{ClSO}_{2} \mathrm{C}_{6} \mathrm{H}_{5},(n-\mathrm{Bu})_{4} \mathrm{~N} \cdot \mathrm{HSO}_{4}, \mathrm{NaOH}, \mathrm{H}_{2} \mathrm{O}, \mathrm{C}_{6} \mathrm{H}_{6}, 25{ }^{\circ} \mathrm{C}$, overnight (2a, 98\%; 2b, 85\%; 2c, 92\%); (b) LDA, THF, $0{ }^{\circ} \mathrm{C}$, $30 \mathrm{~min}$, then $\mathrm{ZnCl}_{2}, 25{ }^{\circ} \mathrm{C}, 30 \mathrm{~min}$; (c) 2bromo-4-methylpyridyne, $\mathrm{Pd}\left[\mathrm{P}\left(\mathrm{C}_{6} \mathrm{H}_{5}\right)_{3}\right]_{4}$, THF, reflux, 4 h (4a, 64\%; 4b, 65\%; 4c, 80\%); (d) $\mathrm{NaOH}, \mathrm{EtOH}, \mathrm{H}_{2} \mathrm{O}$, reflux, 12 h (5a, 85\%; 5b, 93\%; 5c, 87\%).

\section{Scheme 1}

On the other hand, as an extension of our previous work on the use of the Pummerer ${ }^{9}$ reaction in electrophilic cyclizations on the indole 3-position, ${ }^{3 a, 10}$ we describe here the synthesis of indoloquinolizidine 15 from pyridylindole 9. This compound was prepared in 85\% overall yield by palladium(0)-catalyzed cross-coupling reaction of 2-indolylzinc chloride 7 and methyl 2chloropyridine-4-carboxylate, followed by alkaline hydrolysis of the resulting protected pyridylindole $\mathbf{8}$ and reesterification of the corresponding acid. As expected, catalytic hydrogenation of $\mathbf{9}$ stereoselectively afforded the 2,4-cis indolylpiperidine 10. Incorporation of the (phenylsulfinyl)ethyl chain on the piperidine nitrogen was carried out by refluxing a methanolic solution of $\mathbf{1 0}$ and phenyl vinyl sulfoxide. In this way, compound $\mathbf{1 1}$ was obtained in $89 \%$ yield as a mixture of isomers at the sulfur atom. In previous studies ${ }^{3 a}$ we concluded that protection of the indole nitrogen with an electron-withdrawing group constitutes an essential requirement for the success of the closure of the indoloquinolizidine $\mathrm{C}$ ring by electrophilic attack on the indole 3-position of a thionium ion generated by the Pummerer reaction.

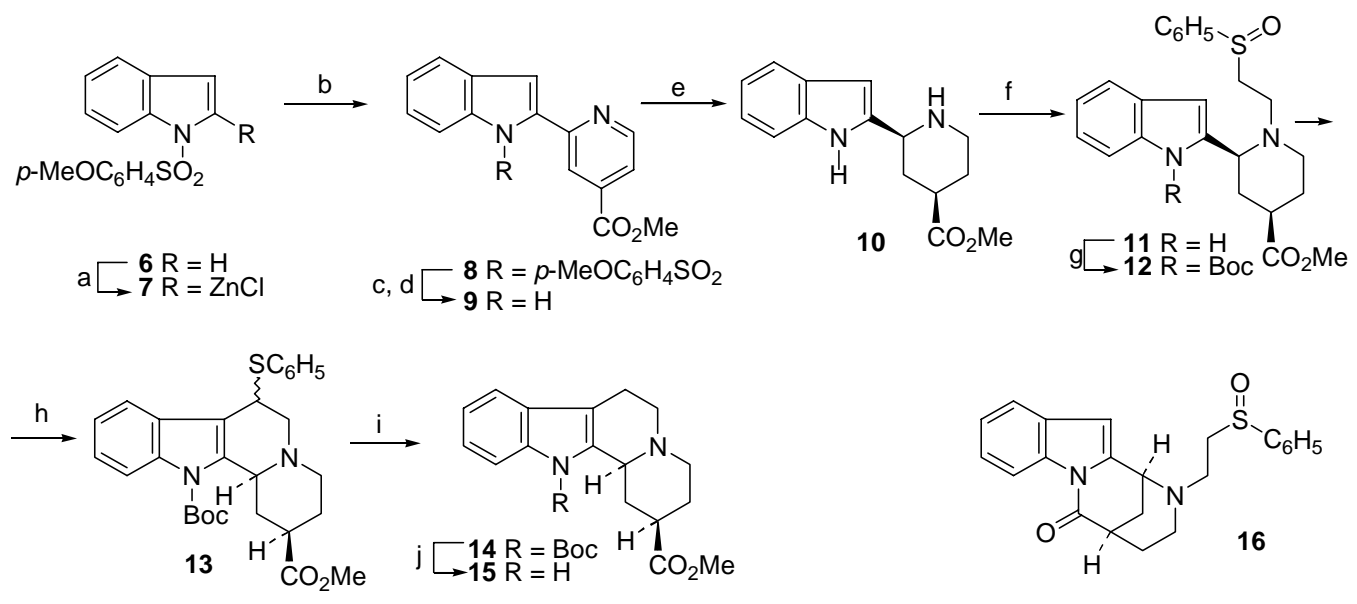

\section{Scheme 2}


Reagents and conditions: (a) LDA, THF, $0{ }^{\circ} \mathrm{C}, 30 \mathrm{~min}$, then $\mathrm{ZnCl}_{2}$; (b) methyl 2-chloropyridine4-carboxylate, $\mathrm{PdCl}_{2}\left(\mathrm{PPh}_{3}\right)_{2}$, DIBAH, THF, reflux (89\%); (c) $\mathrm{NaOH}, \mathrm{EtOH}, \mathrm{H}_{2} \mathrm{O}$, reflux, $12 \mathrm{~h}$, (96\%); (d) $\mathrm{HCl}$, anh. $\mathrm{MeOH}$, reflux, $3 \mathrm{~h} \mathrm{(98 \% );} \mathrm{(e)} \mathrm{H}_{2}, \mathrm{PtO}_{2}, \mathrm{AcOH}$ (65\%); (f) $\mathrm{C}_{6} \mathrm{H}_{5} \mathrm{~S}(\mathrm{O}) \mathrm{CH}=\mathrm{CH}_{2}, \mathrm{MeOH}$, reflux, 24 h (89\%); (g) (Boc) ${ }_{2} \mathrm{O}, \mathrm{DMAP}, \mathrm{CH}_{2} \mathrm{Cl}_{2}, 25{ }^{\circ} \mathrm{C}, 10 \mathrm{~h}$; (85\%) (h) TMSOTf, DIPEA, $\mathrm{CH}_{2} \mathrm{Cl}_{2}, 2{ }^{\circ} \mathrm{C}, 15$ min (84\%); (i) $\mathrm{Bu}_{3} \mathrm{SnH}, \mathrm{AIBN}, \mathrm{C}_{6} \mathrm{H}_{6}$, reflux, $3 \mathrm{~h}$ (75\%); (j) $\mathrm{HCO}_{2} \mathrm{H}, 25{ }^{\circ} \mathrm{C}, 24 \mathrm{~h}(96 \%)$.

This protecting group not only blocks the indole nitrogen but also diminishes the nucleophilic character of the indole nucleus, thus avoiding the formation of both undesired $N$ regioisomers $^{3 \mathrm{a}, 11}$ and byproducts coming from the attack of the indole ring upon the oxysulfonium intermediate ${ }^{12}$ initially formed in the Pummerer reaction. The $N$-Boc derivative 12 was obtained in good yield by treatment of $\mathbf{1 1}$ with di-t-butyl dicarbonate and DMAP in dichloromethane. It is worth mentioning that when this transformation was attempted by treating compound 11 with methyl cyanoformate and sodium hydride, tetracycle $\mathbf{1 6}$ was formed in 50\% yield instead of the desired compound 12. Sulfoxide 12 was subjected to the Pummerer reaction by treatment with TMSOTf and DIPEA in dichloromethane at room temperature. This reaction afforded the desired indoloquinolizidine 13 in $84 \%$ yield as a mixture of epimers at $\mathrm{C}_{7}$. Finally, elimination of the phenylsulfanyl group by treatment of $\mathbf{1 3}$ with tributyltin hydride and AIBN, followed by hydrolysis of the Boc group with formic acid, provided indoloquinolizidine $15^{13}$ in excellent yield.

In conclusion, a convenient route to indolo[2,3-a]quinolizidines involving, as the key steps, a palladium(0)-catalyzed cross-coupling reaction between a 2-indolylzinc derivative and a 2halopyridine and a Pummerer cyclization on the indole 3-position of a 2-(2-piperidyl)indole has been developed.

\section{Experimental Section}

General Procedures. Melting points were determined on a Gallenkamp melting point apparatus and are uncorrected. NMR spectra were recorded on either a Varian Gemini 200 operating at $200 \mathrm{MHz}$ for ${ }^{1} \mathrm{H}$ and $50.3 \mathrm{MHz}$ for ${ }^{13} \mathrm{C}$, or a Varian Gemini 300 operating at $300 \mathrm{MHz}$ for ${ }^{1} \mathrm{H}$ and $75.5 \mathrm{MHz}$ for ${ }^{13} \mathrm{C}$. Chemical shifts are reported in values ppm relative to TMS as internal reference. IR spectra were recorded on a FTIR Perkin-Elmer 1600 spectrometer with samples prepared either as $\mathrm{KBr}$ pellets or thin films on $\mathrm{NaCl}$ salt plates, and only noteworthy absorptions are listed. Thin-layer chromatography was done on $\mathrm{SiO}_{2}$ (silica gel $60 \mathrm{~F}_{254}$, Merck), and the spots were located with aqueous potassium permanganate solution. Column chromatography was carried out on $\mathrm{SiO}_{2}$ (silica gel 60, SDS, 70-200 microns). Flash chromatography was carried out on $\mathrm{SiO}_{2}$ (silica gel 60, SDS, 35-70 microns). All reagents were purchased from Aldrich or Fluka and were used without further purification. Tetrahydrofuran was distilled from sodium/benzophenone. Solvents for chromatography were distilled at atmospheric pressure prior 
to use and dried using standard procedures. All reactions were performed under argon or nitrogen. Drying of the organic extracts during the work-up of reactions was performed over $\mathrm{Na}_{2} \mathrm{SO}_{4}$. Evaporation of solvents was accomplished with a rotatory evaporator. Microanalyses were performed by Centre d’Investigació i Desenvolupament (CSIC), Barcelona.

General procedure for the preparation of protected methoxyindoles 2a-c Tetrabutylammonium hydrogen sulfate $(27 \mathrm{mg}, 78 \mathrm{mmol})$ and a $50 \%$ aqueous $\mathrm{NaOH}$ solution $(8 \mathrm{~mL})$ were added to a vigorously stirred solution of 1a-c $(1.0 \mathrm{~g}, 6.8 \mathrm{mmol})$ in benzene $(20 \mathrm{~mL})$. After $5 \mathrm{~min}$, a solution of benzenesulfonyl chloride $(1.3 \mathrm{~mL}, 10.2 \mathrm{mmol})$ in benzene $(10 \mathrm{~mL})$ was added, and the mixture was stirred at room temperature overnight. The benzene layer was separated, washed with water, dried, and concentrated to give, after flash chromatography (3:2 $\mathrm{CH}_{2} \mathrm{Cl}_{2}$ :hexane), compounds 2a-c.

1-(Benzenesulfonyl)-4-methoxyindole (2a). 98\% yield; mp $77-78{ }^{\circ} \mathrm{C}$ (lit. $\left.{ }^{14} 79-80{ }^{\circ} \mathrm{C}\right) ;{ }^{1} \mathrm{H}$ NMR $\left(\mathrm{CDCl}_{3}, 300 \mathrm{MHz}\right): \delta 3.88(\mathrm{~s}, 3 \mathrm{H}), 6.64(\mathrm{~d}, J=8 \mathrm{~Hz}, 1 \mathrm{H}), 6.77$ (dd, $\left.J=3.7,0.7 \mathrm{~Hz}, 1 \mathrm{H}\right), 7.23(\mathrm{t}$, $J=8 \mathrm{~Hz}, 1 \mathrm{H}), 7.38-7.70(\mathrm{~m}, 4 \mathrm{H}), 7.47$ (d, $J=3.7 \mathrm{~Hz}, 1 \mathrm{H}), 7.60$ (d, $J=8 \mathrm{~Hz}, 1 \mathrm{H}), 7.86(\mathrm{~m}, 1 \mathrm{H})$;

${ }^{13} \mathrm{C} \mathrm{NMR}\left(\mathrm{CDCl}_{3}, 75.4 \mathrm{MHz}\right): \delta 55.4,103.5,106.3,106.4,121.0,124.7,125.7,126.7$ (2 C), 129.2 (2 C), 133.8, 136.0, 138.2, 153.1.

1-(Benzenesulfonyl)-5-methoxyindole (2b). 85\% yield; mp 94-95 ${ }^{\circ} \mathrm{C}$ (lit. ${ }^{15}$ 98-99 $\left.{ }^{\circ} \mathrm{C}\right) ;{ }^{1} \mathrm{H}$ NMR $\left(\mathrm{CDCl}_{3}, 300 \mathrm{MHz}\right): \delta 3.81$ (s, 3H), 6.59 (d, $\left.J=3.6 \mathrm{~Hz}, 1 \mathrm{H}\right), 6.93$ (dd, $\left.J=8.8,2.5 \mathrm{~Hz}, 1 \mathrm{H}\right), 6.96$ (d, $J=2.4 \mathrm{~Hz}, 1 \mathrm{H}), 7.39-7.56(\mathrm{~m}, 3 \mathrm{H}), 7.52(\mathrm{~d}, J=3.6 \mathrm{~Hz}, 1 \mathrm{H}), 7.83-7.90(\mathrm{~m}, 3 \mathrm{H})$; ${ }^{13} \mathrm{C}$ NMR $\left(\mathrm{CDCl}_{3}, 75.4 \mathrm{MHz}\right): \delta$ 55.6, 103.6, 109.4, 113.7, 114.3, 126.6 (2 C), 127.0, 129.1 (2 C), 129.5, 131.7, 133.7, 138.0, 156.4.

1-(Benzenesulfonyl)-6-methoxyindole (2c). 92\% yield; mp 138-139 ${ }^{\circ} \mathrm{C}$ (lit. ${ }^{15} 140-142{ }^{\circ} \mathrm{C}$ ); ${ }^{1} \mathrm{H}$ NMR (CDCl $3,300 \mathrm{MHz}$ ): $\delta 3.87$ (s, $3 \mathrm{H}$ ), 6.58 (dd, $J=3.6,0.6 \mathrm{~Hz}, 1 \mathrm{H}$ ), 6.86 (dd, $J=8.7,2.4$ $\mathrm{Hz}, 1 \mathrm{H}), 7.38$ (d, $J=8.7 \mathrm{~Hz}, 1 \mathrm{H}), 7.42$ (t, $J=8.4 \mathrm{~Hz}, 2 \mathrm{H}), 7.43$ (d, $J=3.6 \mathrm{~Hz}, 1 \mathrm{H}), 7.51(\mathrm{~d}, J=$ $8.4 \mathrm{~Hz}, 1 \mathrm{H}), 7.54$ (d, $J=2.4 \mathrm{~Hz}, 1 \mathrm{H}), 7.87$ (d, $J=8.4 \mathrm{~Hz}, 2 \mathrm{H}) ;{ }^{13} \mathrm{C} \mathrm{NMR}\left(\mathrm{CDCl}_{3}, 75.4 \mathrm{MHz}\right): \delta$ 55.7, 97.9, 109.1, 112.5, 124.4, 125.0, 125.1, 126.6 (2 C), 129.1 (2 C), 133.7, 135.9, 138.3, 157.9.

\section{General procedure for the preparation of methoxy-2-(2-pyridyl)indoles 4a-c}

A solution of LDA (1.34 mL of a $1.5 \mathrm{M}$ solution in cyclohexane, $2.0 \mathrm{mmol}$ ) was slowly added to a solution of indoles 2a-c (500 mg, $1.83 \mathrm{mmol}$ ) in anhydrous THF (3 mL) at $0{ }^{\circ} \mathrm{C}$, and the resulting mixture was stirred for $30 \mathrm{~min}$ at this temperature. Then, a solution of anhydrous $\mathrm{ZnCl}_{2}$ (330 mg, $2.0 \mathrm{mmol}$ ) in THF (4.5 mL) was added, and the stirring was continued for $30 \mathrm{~min}$ at $25{ }^{\circ} \mathrm{C}$. In a separate flask, a solution of 2-bromo-4-methylpyridine (210 $\mathrm{mg}, 1.22 \mathrm{mmol}$ ) in anhydrous THF (1.5 mL) was added to a solution of tetrakis(triphenylphosphine)palladium(0) (141 mg, $0.12 \mathrm{mmol}$ ) in THF (4.5 mL), and the mixture was stirred at $25^{\circ} \mathrm{C}$ for $10 \mathrm{~min}$. The resulting solution was transferred via canula to the solution of methoxyindolylzinc chloride 3a-c prepared above and the mixture was heated at reflux for $4 \mathrm{~h}$, cooled, and poured into saturated 
aqueous $\mathrm{Na}_{2} \mathrm{CO}_{3}$. The aqueous phase was extracted with $\mathrm{Et}_{2} \mathrm{O}$, and the organic extracts were concentrated to give a residue, which was purified by flash chromatography $\left(\mathrm{CH}_{2} \mathrm{Cl}_{2}\right)$.

1-(Benzenesulfonyl)-4-methoxy-2-(4-methyl-2-pyridyl)indole (4a). 64\% yield; IR (KBr): 3450, 1607, 1369, 1103, $784 \mathrm{~cm}^{-1}$; ${ }^{1} \mathrm{H}$ NMR $\left(\mathrm{CDCl}_{3}, 300 \mathrm{MHz}\right): \delta 2.42$ (s, 3H), 3.83 (s, 3H), $6.64(\mathrm{~d}, J=8.0 \mathrm{~Hz}, 1 \mathrm{H}), 6.96(\mathrm{~s}, 1 \mathrm{H}), 7.13(\mathrm{dm}, J=5.2 \mathrm{~Hz}, 1 \mathrm{H}), 7.26$ (t, $J=8.0 \mathrm{~Hz}, 1 \mathrm{H}), 7.32$ (tm, $J=7.8 \mathrm{~Hz}, 1 \mathrm{H}$ ), 7.43 (t, $J=7.8,1.2 \mathrm{~Hz}, 1 \mathrm{H}), 7.48$ (br s, 1H), 7.73 (dm, $J=7.8 \mathrm{~Hz}, 1 \mathrm{H}$ ), 7.77 (d, $J=8.0 \mathrm{~Hz}, 1 \mathrm{H}), 8.52$ (d, $J=5.2 \mathrm{~Hz}, 1 \mathrm{H}) ;{ }^{13} \mathrm{C} \mathrm{NMR}\left(\mathrm{CDCl}_{3}, 75.4 \mathrm{MHz}\right): \delta 21.1,55.4$, 104.5, 108.8, 111.8, 120.7, 123.9, 126.2, 126.7, 127.0 (2 C), 128.6 (2 C), 133.5, 137.3, 139.1, 139.5, 146.5, 148.5, 151.2, 153.1.

1-(Benzenesulfonyl)-5-methoxy-2-(4-methyl-2-pyridyl)indole (4b). 65\% yield; ${ }^{1} \mathrm{H} \quad \mathrm{NMR}$ $\left(\mathrm{CDCl}_{3}, 300 \mathrm{MHz}\right): \delta 2.44$ (s, 3H), 3.80 (s, 3H), 6.80 (d, $\left.J=0.9 \mathrm{~Hz}, 1 \mathrm{H}\right), 6.88$ (d, $J=2.5 \mathrm{~Hz}$, $1 \mathrm{H}), 6.95$ (dd, $J=9.0,2.5 \mathrm{~Hz}, 1 \mathrm{H}), 7.16(\mathrm{dm}, J=5.1 \mathrm{~Hz}, 1 \mathrm{H}), 7.30(\mathrm{tm}, J=7.8 \mathrm{~Hz}, 1 \mathrm{H}), 7.43$ (tt, $J=7.8,1.2 \mathrm{~Hz}, 1 \mathrm{H}), 7.53$ (m, 1H), 7.60 (dm, $J=7.8 \mathrm{~Hz}, 1 \mathrm{H}), 8.08$ (d, $J=9.0 \mathrm{~Hz}, 1 \mathrm{H}), 8.54$ (d, $J$ $=5.1 \mathrm{~Hz}, 1 \mathrm{H}) ;{ }^{13} \mathrm{C} \mathrm{NMR}\left(\mathrm{CDCl}_{3}, 75.4 \mathrm{MHz}\right): \delta 21.1,55.5,103.6,114.1,115.6,117.4,124.2$, 127.0 (2 C), 127.2, 128.2, 128.6 (2 C), 132.6, 133.5, 136.6, 142.2, 146.5, 148.6, 151.0, 157.1.

1-(Benzenesulfonyl)-6-methoxy-2-(4-methyl-2-pyridyl)indole (4c). 80\% yield; IR (film): 3350, 1610, 1371, 1176, 591 $\mathrm{cm}^{-1}$; ${ }^{1} \mathrm{H}$ NMR ( $\left.\mathrm{CDCl}_{3}, 300 \mathrm{MHz}\right): \delta 2.43$ (s, 3H), 3.91 (s, 3H), 6.78 (d, $J=0.9 \mathrm{~Hz}, 1 \mathrm{H}), 6.86$ (dd, $J=8.6,2.3 \mathrm{~Hz}, 1 \mathrm{H}), 7.12(\mathrm{dm}, J=5.2 \mathrm{~Hz}, 1 \mathrm{H}), 7.32$ (d, $J=$ $8.6 \mathrm{~Hz}, 1 \mathrm{H}), 7.33$ (tm, $J=7.5 \mathrm{~Hz}, 1 \mathrm{H}), 7.50$ (tt, $J=7.5,1.5 \mathrm{~Hz}, 1 \mathrm{H}), 7.52(\mathrm{~m}, 1 \mathrm{H}), 7.65(\mathrm{dm}, J=$ $7.5 \mathrm{~Hz}, 1 \mathrm{H}), 7.74$ (d, $J=2.3 \mathrm{~Hz}, 1 \mathrm{H}), 8.51$ (dd, $J=5.2,0.6 \mathrm{~Hz}, 1 \mathrm{H}) ;{ }^{13} \mathrm{C} \mathrm{NMR}\left(\mathrm{CDCl}_{3}, 75.4\right.$ MHz): $\delta$ 21.0, 55.8, 100.7, 113.4, 115.1, 121.7, 123.8, 124.2, 126.8, 126.9 (2 C), 128.5 (2 C), 133.5, 137.0, 139.4, 140.1, 146.4, 148.5, 151.2, 158.3.

\section{General procedure for the preparation of methoxy-2-(2-pyridyl)indoles 5a-c}

A solution of compounds 4a-c (250 mg, $0.66 \mathrm{mmol})$ in EtOH (40 mL) and 10\% aqueous $\mathrm{NaOH}$ ( $4 \mathrm{~mL}$ ) was heated at reflux for $12 \mathrm{~h}$. The resulting mixture was concentrated, and the residue was dissolved in $\mathrm{CH}_{2} \mathrm{Cl}_{2}(15 \mathrm{~mL})$. The organic solution was washed with water and aqueous $\mathrm{Na}_{2} \mathrm{CO}_{3}$, dried, concentrated, and the resulting residue was purified by flash chromatography $\left(\mathrm{CH}_{2} \mathrm{Cl}_{2}\right)$.

4-Methoxy-2-(4-methyl-2-pyridyl)indole (5a). 85\% yield; IR (film): 3162, 1610, 1247, 1105, $771 \mathrm{~cm}^{-1} .{ }^{1} \mathrm{H}$ NMR $\left(\mathrm{CDCl}_{3}, 300 \mathrm{MHz}\right): \delta 2.37$ (s, 3H), $3.98(\mathrm{~s}, 3 \mathrm{H}), 6.50(\mathrm{~d}, J=7.8 \mathrm{~Hz}, 1 \mathrm{H})$, 6.97 (d, $J=5.1 \mathrm{~Hz}, 1 \mathrm{H}), 7.02$ (d, $J=7.8 \mathrm{~Hz}, 1 \mathrm{H}), 7.12$ (t, $J=7.8 \mathrm{~Hz}, 1 \mathrm{H}), 7.14$ (s, 1H), 7.64 (s, 1H), 8.36 (d, $J=5.1 \mathrm{~Hz}, 1 \mathrm{H}), 10.25$ (br s, $1 \mathrm{H}) .{ }^{13} \mathrm{C} \mathrm{NMR}\left(\mathrm{CDCl}_{3}, 75.4 \mathrm{MHz}\right): \delta 20.9,55.1,98.1$, 99.3, 104.7, 120.0, 120.7, 122.9, 123.7, 135.0, 139.0, 148.0, 148.3, 150.2, 153.4; Anal. Calcd for $\mathrm{C}_{15} \mathrm{H}_{14} \mathrm{~N}_{2} \mathrm{O} \cdot 2 / 3 \mathrm{H}_{2} \mathrm{O}$ : C, 71.96; H, 6.18; N, 11.19. Found: C, 71.94; H, 5.75; N, 10.79.

5-Methoxy-2-(4-methyl-2-pyridyl)indole (5b). 93\% yield; mp 115-116 ${ }^{\circ} \mathrm{C}$; IR (film): 3150, 1606, 1225, $801 \mathrm{~cm}^{-1}$; ${ }^{1} \mathrm{H}$ NMR $\left(\mathrm{CDCl}_{3}, 300 \mathrm{MHz}\right): \delta 2.42$ (s, 3H), 3.88 (s, 3H), 6.88 (dd, $J=$ 9.0, $2.3 \mathrm{~Hz}, 1 \mathrm{H}), 6.95(\mathrm{~m}, 1 \mathrm{H}), 7.00(\mathrm{dm}, J=5.1 \mathrm{~Hz}, 1 \mathrm{H}), 7.11$ (d. $J=2.3 \mathrm{~Hz}, 1 \mathrm{H}), 7.25$ (d, $J=$ $9.0 \mathrm{~Hz}, 1 \mathrm{H}), 7.63(\mathrm{~m}, 1 \mathrm{H}), 8.44$ (d, $J=5.1 \mathrm{~Hz}, 1 \mathrm{H}), 10.0$ (br s, $1 \mathrm{H}) ;{ }^{13} \mathrm{C} \mathrm{NMR}\left(\mathrm{CDCl}_{3}, 75.4\right.$ MHz): $\delta$ 21.1, 55.7, 100.0, 102.2, 112.1, 113.6, 120.5, 123.1, 129.4, 131.8, 137, 147.7, 148.7, 
150.2, 154.3; Anal. Calcd for $\mathrm{C}_{15} \mathrm{H}_{14} \mathrm{~N}_{2} \mathrm{O} \cdot 1 / 2 \mathrm{H}_{2} \mathrm{O}$ : C, 72.85; H, 6.11; N, 11.33. Found: C, 72.41; H, 5.92; N, 11.76.

6-Methoxy-2-(4-methyl-2-pyridyl)indole (5c). 87\% yield; mp 145-146 ${ }^{\circ} \mathrm{C}$; IR (KBr): 3418, 1603, 1261, $816 \mathrm{~cm}^{-1} .{ }^{1} \mathrm{H}$ NMR $\left(\mathrm{CDCl}_{3}, 300 \mathrm{MHz}\right): \delta 2.34(\mathrm{~s}, 3 \mathrm{H}), 3.74(\mathrm{~s}, 3 \mathrm{H}), 6.68(\mathrm{~d}, J=$ $2.2 \mathrm{~Hz}, 1 \mathrm{H}), 6.76(\mathrm{dd}, J=8.6,2.2 \mathrm{~Hz}, 1 \mathrm{H}), 6.90(\mathrm{~m}, 1 \mathrm{H}), 6.93(\mathrm{dm}, J=5.1 \mathrm{~Hz}, 1 \mathrm{H}), 7.49(\mathrm{~d}, J=$ $8.6 \mathrm{~Hz}, 1 \mathrm{H}), 7.56$ (br s, $1 \mathrm{H}), 8.39$ (d, $J=5.1 \mathrm{~Hz}, 1 \mathrm{H}), 10.5$ (br s, $1 \mathrm{H}) ;{ }^{13} \mathrm{C} \mathrm{NMR}\left(\mathrm{CDCl}_{3}\right.$, 75.4 MHz): $\delta$ 21.1, 55.4, 94.2, 100.5, 110.4, 120.2, 121.6, 122.6, 123.3, 135.9, 137.6, 147.7, 148.5, 150.5, 157.0; Anal. Calcd for $\mathrm{C}_{15} \mathrm{H}_{14} \mathrm{~N}_{2} \mathrm{O} \cdot 1 / 4 \mathrm{H}_{2} \mathrm{O}$ : C, 74.21; H, 6.02; N, 11.54. Found: C, 74.46; H, 5.83; N, 11.40 .

Methyl 2-[1-(p-methoxybenzenesulfonyl)-2-indolyl]pyridine-4-carboxylate (8). A solution of LDA (51.3 mL, $1.5 \mathrm{M}$ in cyclohexane, $77 \mathrm{mmol}$ ) was slowly added to a solution of sulfonylindole $\mathbf{6}^{16}$ (20 g, $\left.70 \mathrm{mmol}\right)$ in anhydrous THF $(100 \mathrm{~mL})$ at $0{ }^{\circ} \mathrm{C}$, and the resulting mixture was stirred for $30 \mathrm{~min}$ at this temperature. Then, a solution of anhydrous $\mathrm{ZnCl}_{2}(10.5 \mathrm{~g}$, $77 \mathrm{mmol})$ in THF (170 mL) was added, and the stirring was continued for $30 \mathrm{~min}$ at $25^{\circ} \mathrm{C}$. In a separate flask, a solution of dichlorobis(triphenylphosphine)palladium(II) (0.7 g, $1 \mathrm{mmol})$ and DIBAH (2 mL, $1.0 \mathrm{M}$ solution in hexane, $2.0 \mathrm{mmol}$ ) in THF (40 mL) was stirred at $25{ }^{\circ} \mathrm{C}$ for $5 \mathrm{~min}$, and then a solution of methyl 2-chloropyridine-4-carboxylate ${ }^{17}$ (8.6 g, $50 \mathrm{mmol}$ ) in anhydrous THF $(60 \mathrm{~mL})$ was added. The stirring was continued at $25{ }^{\circ} \mathrm{C}$ for $10 \mathrm{~min}$. The resulting solution was transferred via canula to the solution of methoxyindolylzinc chloride 7 prepared above and the mixture was heated at reflux for $4 \mathrm{~h}$, cooled, and poured into saturated aqueous $\mathrm{Na}_{2} \mathrm{CO}_{3}$. The aqueous phase was extracted with $\mathrm{Et}_{2} \mathrm{O}$, and the organic extracts were concentrated to give a residue, which, after purification by flash chromatography $\left(\mathrm{CH}_{2} \mathrm{Cl}_{2}\right)$, afforded pure 2-(2-pyridyl)indole 8 (18.8 g, 89\%): mp 126-127 ${ }^{\circ} \mathrm{C}\left(\mathrm{Et}_{2} \mathrm{O}\right)$; IR (KBr): 1732, 1595, $1371 \mathrm{~cm}^{-1} ;{ }^{1} \mathrm{H} \mathrm{NMR}\left(\mathrm{CDCl}_{3}, 300 \mathrm{MHz}\right): \delta 3.75$ (s, 3H), $3.99(\mathrm{~s}, 3 \mathrm{H}), 6.77(\mathrm{dm}, J=9.1 \mathrm{~Hz}, 2 \mathrm{H})$, 6.90 (s, 1H), 7.26 (td, $J=7.5,1.4 \mathrm{~Hz}, 1 \mathrm{H}), 7.39$ (ddd, $J=8.5,7.5,1.4 \mathrm{~Hz}, 1 \mathrm{H}$ ), 7.49 (dd, $J=7.5$, $0.7 \mathrm{~Hz}, 1 \mathrm{H}), 7.57$ (dm, $J=9.1 \mathrm{~Hz}, 2 \mathrm{H}), 7.90$ (dd, $J=5.1,1.6 \mathrm{~Hz}, 1 \mathrm{H}), 8.20$ (d, $J=8.5 \mathrm{~Hz}, 1 \mathrm{H}$ ), 8.27 (br s, $1 \mathrm{H}), 8.84$ (d, $J=5.1 \mathrm{~Hz}, 1 \mathrm{H}) ;{ }^{13} \mathrm{C} \mathrm{NMR}\left(\mathrm{CDCl}_{3}, 50.3 \mathrm{MHz}\right): \delta$ 52.7, 55.4, 113.8 (2 C), 115.8, 116.3, 121.4, 122.0, 124.4, 125.4, 125.5, 128.4, 129.2 (2 C), 130.3, 136.9, 138.2, 140.2, 149.5, 152.5, 163.5, 165.3; Anal. Calcd for $\mathrm{C}_{22} \mathrm{H}_{18} \mathrm{~N}_{2} \mathrm{O}_{5} \mathrm{~S}$ : C, 62.55; H, 4.30; N, 6.63; S, 7.59. Found: C, 62.23; H, 4.18; N, 6.46; S, 7.60.

Methyl 2-(2-indolyl)pyridine-4-carboxylate (9). A solution of compound 8 (1.8 g, $4.27 \mathrm{mmol}$ ) in $\mathrm{EtOH}(200 \mathrm{~mL})$ and $10 \%$ aqueous $\mathrm{NaOH}(25 \mathrm{~mL})$ was heated at reflux for $12 \mathrm{~h}$, cooled, concentrated, and the residue treated with $10 \%$ aqueous $\mathrm{H}_{2} \mathrm{SO}_{4}(10 \mathrm{~mL})$. The resulting precipitate was filtered, washed with water, and dried to give acid 2-(2-indolyl)pyridine-4carboxylic (0.98 g, 96\%) as a white solid: mp 286-289 ${ }^{\circ} \mathrm{C}\left(\mathrm{H}_{2} \mathrm{O}\right)$; IR $(\mathrm{KBr}): 3300,1713,1620$ $\mathrm{cm}^{-1}$; ${ }^{1} \mathrm{H}$ NMR (DMSO, $200 \mathrm{MHz}$ ): $\delta 7.01$ (t, $\left.J=7.8 \mathrm{~Hz}, 1 \mathrm{H}\right), 7.13$ (t, $\left.J=7.8 \mathrm{~Hz}, 1 \mathrm{H}\right), 7.25$ (s, $1 \mathrm{H}), 7.46$ (d, $J=7.8 \mathrm{~Hz}, 1 \mathrm{H}), 7.58$ (d, $J=7.8 \mathrm{~Hz}, 1 \mathrm{H}), 7.69$ (d, $J=4.8 \mathrm{~Hz}, 1 \mathrm{H}), 8.35$ (s, 1H), 8.79 (d, $J=4.8 \mathrm{~Hz}, 1 \mathrm{H}$ ), 11.8 (br s, $1 \mathrm{H}$ ); ${ }^{13} \mathrm{C}$ NMR (DMSO, 50.3 MHz): 101.8, 112.4, 119.0, 119.9, 121.2 (2 C), 123.0, 128.6, 136.7, 137.7, 139.5, 150.6, 151.7, 166.5; Anal. Calcd for $\mathrm{C}_{14} \mathrm{H}_{10} \mathrm{~N}_{2} \mathrm{O}_{2} \cdot 1 / 2 \mathrm{H}_{2} \mathrm{O}$ : C, 68.01; H, 4.48; N, 11.33. Found: C, 68.36; H, 4.05; N, 11.33. A 
solution of this acid $(0.76 \mathrm{~g}, 3.2 \mathrm{mmol})$ in a saturated $\mathrm{HCl}$ methanolic solution $(100 \mathrm{~mL})$ was heated at reflux under stirring for $3 \mathrm{~h}$. The solution was cooled and concentrated under reduced pressure, and the resulting residue was taken up with saturated aqueous $\mathrm{NaHCO}_{3}(10 \mathrm{~mL})$. The precipitate was filtered and dried to give compound 9 (0.79 g, 98\%) as a white solid: mp 160$161{ }^{\circ} \mathrm{C}\left(\mathrm{CH}_{2} \mathrm{Cl}_{2}\right)$; IR (film): 3370, $1719 \mathrm{~cm}^{-1} ;{ }^{1} \mathrm{H}$ NMR $\left(\mathrm{CDCl}_{3}, 300 \mathrm{MHz}\right): \delta 4.01$ (s, 3H), 7.14 (ddd, $J=8.0,7.0,1.0 \mathrm{~Hz}, 1 \mathrm{H}$ ), 7.15 (s, 1H), 7.24 (ddd, $J=8.0,7.0,1.4 \mathrm{~Hz}, 1 \mathrm{H}$ ), 7.43 (d, $J=8.0$ $\mathrm{Hz}, 1 \mathrm{H}), 7.68$ (d, $J=8.0 \mathrm{~Hz}, 1 \mathrm{H}), 7.70$ (dd, $J=4.8,1.6 \mathrm{~Hz}, 1 \mathrm{H}), 8.33$ (dd, $J=1.6,1.0 \mathrm{~Hz}, 1 \mathrm{H}$ ), 8.70 (dd, $J=4.8,1.0 \mathrm{~Hz}, 1 \mathrm{H}), 9.55$ (s, $1 \mathrm{H}) ;{ }^{13} \mathrm{C} \mathrm{NMR}\left(\mathrm{CDCl}_{3}, 50.3 \mathrm{MHz}\right): \delta 52.7,101.7,111.4$, 119.3, 120.2, 120.7, 121.3, 123.5, 128.9, 135.9, 136.7, 137.9, 149.7, 151.3, 165.4; Anal. Calcd for $\mathrm{C}_{15} \mathrm{H}_{12} \mathrm{~N}_{2} \mathrm{O}_{2}$ : C, 71.42; H, 4.79; N, 11.11. Found: C, 71.03; H, 4.79; N, 10.94 .

Methyl cis-2-(2-indolyl)piperidine-4-carboxylate (10). A solution of pyridylindole 9 (4.0 g, $16 \mathrm{mmol})$ in glacial acetic acid $(50 \mathrm{~mL})$ was shaken at $25^{\circ} \mathrm{C}$ under hydrogen in the presence of $\mathrm{PtO}_{2}(600 \mathrm{mg})$. The catalyst was removed by filtration, and the solvent was evaporated. The residue was dissolved in $\mathrm{CH}_{2} \mathrm{Cl}_{2}$ and the solution was washed with saturated aqueous $\mathrm{Na}_{2} \mathrm{CO}_{3}$, dried, filtered, and concentrated under reduced pressure. Column chromatography (95:3:2 $\mathrm{Et}_{2} \mathrm{O}$ :acetone:DEA) of the residue afforded pure piperidine 10 (2.68 g, 65\%): $\mathrm{mp} 116-117^{\circ} \mathrm{C}$ $\left(\mathrm{Et}_{2} \mathrm{O}\right) ;{ }^{1} \mathrm{H}$ NMR $\left(\mathrm{CDCl}_{3}, 300 \mathrm{MHz}\right): \delta 1.63$ (qd, $\left.J=12.5,4.2 \mathrm{~Hz}, 1 \mathrm{H}\right), 1.68-1.80$ (m, $\left.2 \mathrm{H}\right), 1.98$ (dm, $J=13.2 \mathrm{~Hz}, 1 \mathrm{H}), 2.29(\mathrm{dm}, J=12.9 \mathrm{~Hz}, 1 \mathrm{H}), 2.60$ (tt, $J=12.3,3.8 \mathrm{~Hz}, 1 \mathrm{H}), 2.83(\mathrm{td}, J=$ 12.5, $2.7 \mathrm{~Hz}, 1 \mathrm{H}$ ), 3.26 (ddd, $J=12.5,4.1,2.4 \mathrm{~Hz}, 1 \mathrm{H}$ ), 3.70 (s, 3H), 3.91 (dd, $J=11.3,2.5 \mathrm{~Hz}$, 1H), 6.35 (d, $J=1.0 \mathrm{~Hz}, 1 \mathrm{H}), 7.03-7.16$ (m, 2H), 7.30 (d, $J=8.0 \mathrm{~Hz}, 1 \mathrm{H}), 7.55$ (d, $J=7.7 \mathrm{~Hz}$, 1H), 8.85 (s, $1 \mathrm{H}) ;{ }^{13} \mathrm{C} \mathrm{NMR}\left(\mathrm{CDCl}_{3}, 50.3 \mathrm{MHz}\right): \delta 28.6,35.1,41.7,45.9,51.7,54.3,98.6,110.7$, 119.5, 120.2, 121.5, 127.9, 135.6, 140.7, 175.0; Anal. Calcd for $\mathrm{C}_{15} \mathrm{H}_{18} \mathrm{~N}_{2} \mathrm{O}_{2} \cdot 1 / 2 \mathrm{H}_{2} \mathrm{O}$ : C, 67.40; H, 7.16; N, 10.85. Found: C, 67.00; H, 7.20; N, 10.54 .

Methyl cis-2-(2-indolyl)-1-[2-(phenylsulfinyl)ethyl]piperidine-4-carboxylate (11). A solution of indolylpiperidine 10 (1.11 g, $4.3 \mathrm{mmol}$ ) and phenyl vinyl sulfoxide (980 $\mathrm{mg}, 6.4 \mathrm{mmol}$ ) in $\mathrm{MeOH}(15 \mathrm{~mL})$ was heated at reflux for $24 \mathrm{~h}$. The mixture was cooled, concentrated, and the residue was chromatographed (20:1 $\left.\mathrm{Et}_{2} \mathrm{O}: \mathrm{MeOH}\right)$ to give two epimeric sulfoxides 11 (1.57 g, 89\%). Major epimer (1,15 g, higher Rf): mp 162-163 ${ }^{\circ} \mathrm{C}\left(\mathrm{CH}_{2} \mathrm{Cl}_{2}\right)$; IR (film): $1731 \mathrm{~cm}^{-1} ;{ }^{1} \mathrm{H}$ NMR (CDCl 300 MHz): $\delta$ 1.89-2.12 (m, 3H), 2.18 (ddd, $J=13.2,4.1,2.9 \mathrm{~Hz}, 1 \mathrm{H}$ ), 2.25 (ddd, $J=12.3,11.5,2.8 \mathrm{~Hz}, 1 \mathrm{H}$ ), 2.37 (ddd, $J=13.1,6.4,2.7 \mathrm{~Hz}, 1 \mathrm{H}), 2.55$ (tt, $J=12.3,4.1 \mathrm{~Hz}, 1 \mathrm{H}$ ), 2.73-2.90 (m, 2H), 3.13 (ddd, $J=13.1,9.7,7.7 \mathrm{~Hz}, 1 \mathrm{H}$ ), 3.34 (dt, $J=11.5,3.3 \mathrm{~Hz}, 1 \mathrm{H}$ ), 3.58 (dd, $J=11.4,2.9 \mathrm{~Hz}, 1 \mathrm{H}), 3.66$ (s, 3H), 6.32 (s, 1H), 7.06 (ddd, $J=8.0,7.0,1.0 \mathrm{~Hz}, 1 \mathrm{H}$ ), 7.15 (ddd, $J$ = 8.0, 7.0, 1,0 Hz, 1H), 7.43-7.62 (m, 7H), $9.96(\mathrm{~s}, 1 \mathrm{H}) ;{ }^{13} \mathrm{C} \mathrm{NMR}\left(\mathrm{CDCl}_{3}, 75.4 \mathrm{MHz}\right): \delta 27.8$, 37.7, 41.6, 47.1, 51.3, 51.7, 54.8, 61.5, 100.2, 111.7, 119.3, 119.7, 121.3, 124.0, 128.1, 129.2 (2 C), 130.9, 136.7, 140.1, 143.9, 174.7; Anal. Calcd for $\mathrm{C}_{23} \mathrm{H}_{26} \mathrm{~N}_{2} \mathrm{O}_{3} \mathrm{~S}$ : C, 67.29; H, 6.38; N, 6.82; S, 7.81. Found: C, 67.19; H, 6.54; N, 6.78; S, 7.65. Minor isomer (0.42 g, lower Rf): mp 120$121{ }^{\circ} \mathrm{C}\left(\mathrm{Et}_{2} \mathrm{O}\right)$; IR (KBr): $1731 \mathrm{~cm}^{-1} ;{ }^{1} \mathrm{H} \mathrm{NMR}\left(\mathrm{CDCl}_{3}, 300 \mathrm{MHz}\right): \delta 1.77$ (qd, $J=12.3,3.9 \mathrm{~Hz}$, 1H), 1.92 (t, $J=12.2 \mathrm{~Hz}, 1 \mathrm{H}$ ), 1.90 (masked, 1H), 2.14 (ddd, $J=13.2,3.8,2.8 \mathrm{~Hz}, 1 \mathrm{H}$ ), 2.28 (ddd, $J=12.3,11.6,2.6 \mathrm{~Hz}, 1 \mathrm{H}), 2.47$ (tt, $J=12.3$, $3.8 \mathrm{~Hz}, 1 \mathrm{H}), 2.52-2.92$ (m, 4H), 3.21 (dt, $J=$ 11.6, 3.3 Hz, 1H), 3.47 (dd, $J=11.4,2.8 \mathrm{~Hz}, 1 \mathrm{H}$ ), 3.66 (s, 3H), 6.30 (s, 1H), 7.09 (ddd, $J=8.0$, 
7.0, $1.0 \mathrm{~Hz}, 1 \mathrm{H}), 7.17$ (ddd, $J=8.0,7.0,1.0 \mathrm{~Hz}, 1 \mathrm{H}), 7.25-7.39(\mathrm{~m}, 6 \mathrm{H}), 7.53$ (d, $J=8.0 \mathrm{~Hz}$, 1H), 9.02 (s, 1H); ${ }^{13} \mathrm{C} \mathrm{NMR}\left(\mathrm{CDCl}_{3}, 50.3 \mathrm{MHz}\right): \delta$ 27.6, 35.6, 41.3, 48.1, 51.7, 52.8, 54.8, 61.0, 100.8, 111.3, 119.5, 120.1, 121.6, 123.7 (2 C), 127.9, 128.9 (2 C), 130.7, 136.2, 139.3, 143.3, 174.8 .

Methyl cis-2-[1-(tert-butoxycarbonyl)-2-indolyl]-1-[2-(phenylsulfinyl)ethyl]-piperidine-4-carboxylate (12). A mixture of 11 (200 mg, $0.49 \mathrm{mmol}$, mixture of epimers), DMAP (10 mg, $0.08 \mathrm{mmol}$ ), and di-tert-butyl dicarbonate (229 mg, $1.05 \mathrm{mmol})$ in anhydrous $\mathrm{CH}_{2} \mathrm{Cl}_{2}(4 \mathrm{~mL})$ was stirred at $25{ }^{\circ} \mathrm{C}$ for $10 \mathrm{~h}$. The resulting mixture was concentrated and the residue was chromatographed (12:1 $\mathrm{Et}_{2} \mathrm{O}: \mathrm{MeOH}$ ) to give pure compound 12 as a mixture of epimers (211 mg, 85\%). Higher Rf epimer: mp 131-132 ${ }^{\circ} \mathrm{C}\left(\mathrm{Et}_{2} \mathrm{O}\right)$; IR (KBr): $1730 \mathrm{~cm}^{-1} ;{ }^{1} \mathrm{H} \mathrm{NMR}\left(\mathrm{CDCl}_{3}, 300 \mathrm{MHz}\right): \delta 1.71$ (s, 9H), 1.66-1.84 (m, 2H), $1.92(\mathrm{dm}, J=12.3 \mathrm{~Hz}, 1 \mathrm{H}), 2.28$ (dm, $J=12.5 \mathrm{~Hz}, 1 \mathrm{H}), 2.43$ (td, $J=$ 12.2, $2.5 \mathrm{~Hz}, 1 \mathrm{H}), 2.45-2.59(\mathrm{~m}, 2 \mathrm{H}), 2.65-2.70(\mathrm{~m}, 2 \mathrm{H}), 3.12$ (dt, $J=13.4,7.9 \mathrm{~Hz}, 1 \mathrm{H}), 3.21$ (dt, $J=12.3,3.0 \mathrm{~Hz}, 1 \mathrm{H}$ ), 3.67 (s, 3H), 4.37 (dd, $J=7.4,1.1 \mathrm{~Hz}, 1 \mathrm{H}), 6.68$ (s, 1H), 7.27 (ddd, $J=8.4$, 7.4. $1.4 \mathrm{~Hz}, 1 \mathrm{H}), 7.29-7.35$ (m, 3H), 7.42-7.49 (m, 3H), 8.04 (d, $J=8.4 \mathrm{~Hz}, 1 \mathrm{H})$; ${ }^{13} \mathrm{C} \mathrm{NMR}$ $\left(\mathrm{CDCl}_{3}, 75.4 \mathrm{MHz}\right): \delta$ 26.8, 28.2, 34.3, 41.6, 45.9, 51.6, 52.7, 55.4, 58.8, 84.2, 107.9, 115.3, 120.5, 122.6, 123.5 (2 C), 123.7, 128.8 (2 C), 130.5, 136.2, 142.4, 143.7, 150.3, 174.8; Anal. Calcd for $\mathrm{C}_{28} \mathrm{H}_{34} \mathrm{~N}_{2} \mathrm{O}_{5} \mathrm{~S}$ : C, 65.86; H, 6.71; N, 5.49; S, 6.28. Found: C, 66.01; H, 6.85; N, 5.50; $\mathrm{S}$, 6.16. Lower Rf epimer: mp 113-114 ${ }^{\circ} \mathrm{C}$ (hexane-Et $\mathrm{E}_{2} \mathrm{O}$ ); IR (KBr): $1731 \mathrm{~cm}^{-1}$; ${ }^{1} \mathrm{H}$ NMR $\left(\mathrm{CDCl}_{3}, 300 \mathrm{MHz}\right.$ ): $\delta 1.67$ (s, 9H), 1.68 (masked, $1 \mathrm{H}$ ), 1.80 (qd, $J=12.2,3.8 \mathrm{~Hz}, 1 \mathrm{H}$ ), 1.98 $(\mathrm{dm}, J=12.2 \mathrm{~Hz}, 1 \mathrm{H}), 2.29(\mathrm{dm}, J=12.2 \mathrm{~Hz}, 1 \mathrm{H}), 2.33(\mathrm{td}, J=12.2,2.5 \mathrm{~Hz}, 1 \mathrm{H}), 2.47(\mathrm{tt}, J=$ 12.3, $2.5 \mathrm{~Hz}, 1 \mathrm{H}), 2.58$ (m, 1H), 2.77-2.91 (m, 3H), 3.24 (dt, $J=11.6,3.3 \mathrm{~Hz}, 1 \mathrm{H}), 3.65$ (s, $3 \mathrm{H})$, 4.25 (dd, $J=11.0,2.5 \mathrm{~Hz}, 1 \mathrm{H}), 6.63$ (s, 1H), 7.20-7.30 (m, 5H), 7.35-7.40 (m, 2H), 7.45 (d, $J=$ $8.0 \mathrm{~Hz}, 1 \mathrm{H}), 8.00(\mathrm{~d}, J=8.0 \mathrm{~Hz}, 1 \mathrm{H}) ;{ }^{13} \mathrm{C} \mathrm{NMR}\left(\mathrm{CDCl}_{3}, 75.4 \mathrm{MHz}\right): \delta 27.7,28.1,36.3,41.4$, 47.1, 51.5, 52.8, 54.4, 59.2, 84.2, 107.9, 115.4, 120.3, 122.6, 123.5 (2 C), 128.7 (2 C), 130.6, 136.0, 142.9, 143.1, 150.1, 174.6.

5-Oxo-1-[2-(phenylsulfinyl)ethyl]-2,6-methano[1,4]diazocino[1,2-a]indole (16). Methyl cyanoformiate (66 mg, $0.78 \mathrm{mmol}$ ) was added to a solution of compound 11 (320 mg, $0.78 \mathrm{mmol})$ and TMEDA (100 $\mu \mathrm{L})$ in anhydrous THF (5 mL) containing $\mathrm{NaH}$ (100 mg of a 45-50\% dispersion in mineral oil) and the mixture was stirred at $25{ }^{\circ} \mathrm{C}$. After $8 \mathrm{~h}$, water was added and the mixture was extracted with $\mathrm{CH}_{2} \mathrm{Cl}_{2}$. The combined organic extracts were dried and concentrated to give a residue, which, after purification by column chromatography (gradient from $\mathrm{Et}_{2} \mathrm{O}$ to 10:1 $\mathrm{Et}_{2} \mathrm{O}: \mathrm{MeOH}$ ), afforded pure diazocinoindole 16 (150 mg, 50\%) as an oil: IR (film): 1704, $1454 \mathrm{~cm}^{-1} ;{ }^{1} \mathrm{H}$ NMR $\left(\mathrm{CDCl}_{3}\right.$, $300 \mathrm{MHz}$ ): $\delta$ 2.02-2.06 (m, 3H), 2.11 (dt, $J=12.9,3.0 \mathrm{~Hz}, 1 \mathrm{H}), 2.29$ (dt, $J=12.9,3.0 \mathrm{~Hz}, 1 \mathrm{H}$ ), 2.62-2.74 (m, 2H), $2.78(\mathrm{~m}, 1 \mathrm{H}), 2.92-3.60(\mathrm{~m}, 3 \mathrm{H}), 3.92(\mathrm{t}, J=3.0 \mathrm{~Hz}, 1 \mathrm{H}), 6.16(\mathrm{~s}, 1 \mathrm{H}), 7.23-$ 7.34 (m, 2H), 7.47-7.60 (m, 4H), $7.68(\mathrm{~m}, 2 \mathrm{H}), 8.43$ (dd, $J=7.6,1.5 \mathrm{~Hz}, 1 \mathrm{H}) ;{ }^{13} \mathrm{C} \mathrm{NMR}\left(\mathrm{CDCl}_{3}\right.$, 75.4 MHz): $\delta$ 29.1, 31.7, 38.0, 46.0, 47.6, 50.8, 54.7, 107.3, 116.3, 120.4, 124.0, 124.0 (2 C), 124.8, 129.2 (2 C), 131.0, 133.4, 134.5, 143.8, 171.7; Anal. Calcd for $\mathrm{C}_{22} \mathrm{H}_{22} \mathrm{~N}_{2} \mathrm{O}_{2} \mathrm{~S} \cdot 1 / 2 \mathrm{H}_{2} \mathrm{O}$ : C, 69.37; H, 6.55; N, 6.74; S, 7.72. Found: C, 69.09; H, 6.42; N, 6.77; S, 7.82.

Methyl cis-12-(tert-Butoxycarbonyl)-7-(phenylsulfanyl)indolo[2,3-a]quinolizidine-2-carboxylate (13). TMSOTf (305 $\mu \mathrm{L}, 1.7 \mathrm{mmol}$ ) was added to a solution of sulfoxide 12 (190 mg, $0.37 \mathrm{mmol}$, mixture of 
epimers) and DIPEA (300 $\mu \mathrm{L}, 1.7 \mathrm{mmol})$ in anhydrous $\mathrm{CH}_{2} \mathrm{Cl}_{2}(1.5 \mathrm{~mL})$, and the mixture was stirred at $25{ }^{\circ} \mathrm{C}$ for $15 \mathrm{~min}$. The solution was poured into aqueous $\mathrm{NaHCO}_{3}$, and the aqueous layer was extracted with $\mathrm{CH}_{2} \mathrm{Cl}_{2}$. The combined organic extracts were dried and concentrated to afford a residue, which, after chromatography (1:1 AcOEt:hexane), gave two epimeric indoloquinolizidines 13 (155 mg, 84\%). Higher Rf epimer ( $\alpha-\mathrm{SC}_{6} \mathrm{H}_{5}, 105 \mathrm{mg}$ 57\%): mp 167-168 ${ }^{\circ} \mathrm{C}\left(\mathrm{Et}_{2} \mathrm{O}\right)$; IR (KBr): $1731 \mathrm{~cm}^{-1} ;{ }^{1} \mathrm{H}$ NMR $\left(\mathrm{CDCl}_{3}, 300 \mathrm{MHz}\right): \delta 1.60-1.87(\mathrm{~m}, 3 \mathrm{H}), 1.68(\mathrm{~s}, 9 \mathrm{H})$, 2.22 (dm, $J=13.5 \mathrm{~Hz}, 1 \mathrm{H}), 2.67$ (tt, $J=12.2,3.8 \mathrm{~Hz}, 1 \mathrm{H}$ ), 2.86 (dd, $J=12.2,2.7 \mathrm{~Hz}, 1 \mathrm{H}$ ), 3.143.30 (m, 2H), 3.51 (dd, $J=12.2,3.8 \mathrm{~Hz}, 1 \mathrm{H}$ ), 3.66 (s, 3H), 4.50 (dd, $J=11.0,2.0 \mathrm{~Hz}, 1 \mathrm{H}$ ), 4.61 (dd, $J=3.8,2.7 \mathrm{~Hz}, 1 \mathrm{H}), 7.22-7.38$ (m, 5H), 7.54 (d, $J=8.0 \mathrm{~Hz}, 2 \mathrm{H}), 7.65$ (dd, $J=7.3,1.5 \mathrm{~Hz}$, $1 \mathrm{H}), 8.13$ (dd, $J=7.2,1.3 \mathrm{~Hz}, 1 \mathrm{H}) ;{ }^{13} \mathrm{C} \mathrm{NMR}\left(\mathrm{CDCl}_{3}, 75.4 \mathrm{MHz}\right): \delta 23.2,27.9,28.2,41.8,42.5$, 50.1, 51.7, 53.9, 56.2, 84.2, 114.3, 115.8, 119.0, 122.8, 124.3, 127.1, 127.8, 129.0 (2 C), 131.9 (2 C), 136.0, 136.6, 138.4, 149.8, 174.9; Anal. Calcd for $\mathrm{C}_{28} \mathrm{H}_{32} \mathrm{~N}_{2} \mathrm{O}_{4} \mathrm{~S}$ : C, 68.27; H, 6.55; N, 5.69; S, 6.51. Found: C, 68.20; H, 6.68; N, 5.62; S, 6.35. Lower Rf epimer ( $\left.\beta-\mathrm{SC}_{6} \mathrm{H}_{5}, 50 \mathrm{mg} 27 \%\right)$ : oil; IR (film): $1731 \mathrm{~cm}^{-1}$; ${ }^{1} \mathrm{H} \mathrm{NMR}\left(\mathrm{CDCl}_{3}, 300 \mathrm{MHz}\right): \delta 1.38$ (ddd, $J=12.0,12.0,10.5 \mathrm{~Hz}, 1 \mathrm{H}$ ), 1.67 (s, 9H), 1.75-1.90 (m, 2H), 2.40 (dm, $J=12.0 \mathrm{~Hz}, 1 \mathrm{H}), 2.57$ (tt, $J=12.0,4.2 \mathrm{~Hz}, 1 \mathrm{H}), 2.73$ (td, $J=12.0,3.5 \mathrm{~Hz}, 1 \mathrm{H}), 3.02-3.10$ (m, 2H), 3.20 (dd, $J=12.0,4.3 \mathrm{~Hz}, 1 \mathrm{H}), 3.67$ (s, $3 \mathrm{H}), 3.85$ (d, $J=10.5$ Hz, 1H), 4.43 (m, 1H), 7.22-7.30 (m, 5H), 7.42-7.47 (m, 2H), 7.77 (dd, $J=7.3,1.5$ $\mathrm{Hz}, 1 \mathrm{H}), 8.09$ (dd, $J=7.2,1.3 \mathrm{~Hz}, 1 \mathrm{H}) ;{ }^{13} \mathrm{C} \mathrm{NMR}\left(\mathrm{CDCl}_{3}, 75.4 \mathrm{MHz}\right): \delta 26.0,28.1,31.6,41.9$, 42.6, 51.7, 54.2, 55.7, 58.7, 84.2, 115.4, 115.8, 119.6, 122.8, 124.2, 127.2, 127.9, 128.8 (2 C), 132.8 (2 C), 135.2, 136.8, 138.4, 150.0, 174.9.

Methyl cis-12-(tert-butoxycarbonyl)indolo[2,3-a]quinolizidine-2-carboxylate (14). A mixture of sulfide 13 (180 mg, $0.37 \mathrm{mmol}$, mixture of epimers), $\mathrm{Bu}_{3} \mathrm{SnH}$ (300 $\mu \mathrm{L}, 1.12 \mathrm{mmol}$ ), and AIBN in benzene $(20 \mathrm{~mL})$ was heated at reflux for $3 \mathrm{~h}$. The mixture was concentrated, and the residue was purified by column chromatography $\left(\mathrm{Et}_{2} \mathrm{O}\right)$ affording pure indoloquinolizidine 14 (105 mg, 75\%): mp 148-149 ${ }^{\circ} \mathrm{C}$ (hexane-Et $2 \mathrm{O}$ ); IR (film): $1729 \mathrm{~cm}^{-1} ;{ }^{1} \mathrm{H}$ NMR $\left(\mathrm{CDCl}_{3}, 300\right.$ MHz): $\delta 1.65$ (ddd, $J=12.1,12.1,10.9 \mathrm{~Hz}, 1 \mathrm{H}), 1.68$ (s, 9H), 1.79 (dm, $J=12.1 \mathrm{~Hz}, 1 \mathrm{H}), 1.90$ (qd, $J=12.1,4.2 \mathrm{~Hz}, 1 \mathrm{H}), 2.42(\mathrm{dm}, J=12.6 \mathrm{~Hz}, 1 \mathrm{H}$ ), 2.66 (tt, $J=12.1,4.1 \mathrm{~Hz}, 1 \mathrm{H}$ ), 2.73-2.85 (m, 3H), 2.94 (td, $J=12.6,3.3 \mathrm{~Hz}, 1 \mathrm{H}), 3.12-3.19$ (m, 1H), 3.20 (ddd, $J=12.6,4.1,2.2 \mathrm{~Hz}, 1 \mathrm{H}$ ), 3.68 (s, 3H), 4.10 (br d, $J=10.9$ Hz, 1H), 7.18-7.30 (m, 2H), 7.40 (dd, $J=7.0,1.0 \mathrm{~Hz}, 1 \mathrm{H}$ ), 8.10 (dd, $J=7.4,1.0 \mathrm{~Hz}, 1 \mathrm{H}) ;{ }^{13} \mathrm{C} \mathrm{NMR}\left(\mathrm{CDCl}_{3}, 75.4 \mathrm{MHz}\right): \delta 22.2,24.9,28.2,30.4,42.0$, 46.7, 51.7, 54.4, 58.1, 83.8, 115.5, 116.0, 117.9, 122.6, 124.0, 129.1, 136.2, 136.7, 150.2, 175.1; Anal. Calcd for $\mathrm{C}_{22} \mathrm{H}_{28} \mathrm{~N}_{2} \mathrm{O}_{4} \cdot 3 / 2 \mathrm{H}_{2} \mathrm{O}$ : C, 64.22; H, 7.46; N, 6.80. Found: C, 64.52; H, 7.16; N, 6.80.

Methyl cis-indolo[2,3-a]quinolizidine-2-carboxylate (15). Compound 14 (65 mg, $0.17 \mathrm{mmol}$ ) was dissolved in formic acid $(10 \mathrm{~mL})$, and the solution was stirred at $25{ }^{\circ} \mathrm{C}$ for $24 \mathrm{~h}$. The resulting mixture was concentrated, and the residue dissolved in $\mathrm{CH}_{2} \mathrm{Cl}_{2}$. The organic solution was washed with $10 \%$ aqueous $\mathrm{Na}_{2} \mathrm{CO}_{3}$, dried, and concentrated to give a residue, which was chromatographed (9:1 Et $2 \mathrm{O}: \mathrm{MeOH})$ affording pure compound $15^{13}$ (46 mg, 96\%): ${ }^{1} \mathrm{H}$ NMR $\left(\mathrm{CDCl}_{3}, 300 \mathrm{MHz}\right): \delta 1.71$ (q, $\left.J=12.3 \mathrm{~Hz}, 1 \mathrm{H}\right), 1.89$ (qd, $\left.J=12.3,4.2 \mathrm{~Hz}, 1 \mathrm{H}\right), 2.03(\mathrm{dm}, J=$ $12.3 \mathrm{~Hz}, 1 \mathrm{H}), 2.33-2.44$ (m, 2H), 2.51 (tt, $J=12.3,3.8 \mathrm{~Hz}, 1 \mathrm{H}), 2.60$ (td, $J=11.0,4.7 \mathrm{~Hz}, 1 \mathrm{H})$, $2.71(\mathrm{dm}, J=14.4 \mathrm{~Hz}, 1 \mathrm{H}), 2.92-3.14(\mathrm{~m}, 3 \mathrm{H}), 3.20$ (dd, $J=11.5,1.8 \mathrm{~Hz}, 1 \mathrm{H}), 3.71$ (s, $3 \mathrm{H})$, 
7.07-7.13 (m, 2H), 7.25 (d, $J=7.2 \mathrm{~Hz}, 1 \mathrm{H}), 7.46$ (d, $J=7.4 \mathrm{~Hz}, 1 \mathrm{H}), 8.01$ (br s, $1 \mathrm{H}) ;{ }^{13} \mathrm{C}$ NMR $\left(\mathrm{CDCl}_{3}, 75.4 \mathrm{MHz}\right): \delta 21.6,28.1,32.2,41.4,51.9,52.9,54.7,59.1,108.3,110.8,118.1,119.3$, $121.4,127.2,134.1,136.0,175.0$.

\section{Acknowledgements}

This work was supported by the DGICYT, Spain (BQU2000-0651), and the CUR, Generalitat de Catalunya (2001SGR-0084). Thanks are also due to the DGICYT for providing a postdoctoral fellowship to G. P.

\section{References and Notes}

1. (a) Kisakürek, M. V.; Leeuwenberg, A. J. M.; Hesse, M. In Alkaloids: Chemical and Biological Perspectives; Pelletier, S. W., Ed.; J. Wiley: New York, 1983; Vol. 1, Chapter 5. (b) Atta-ur-Rahman; Basha, A. Biosynthesis of Indole Alkaloids; Clarendon Press: Oxford, 1983.

2. (a) Brown, R. T. In Indoles, The Monoterpenoid Indole Alkaloids; Saxton, J. E., Ed. In The Chemistry of Heterocyclic Compounds; Weissberger, A.; Taylor, E. C., Eds.; J. Wiley: New York, 1983; Vol. 25, Part 4, Ch. 3. (b) Saxton, J. E. Ibid. Ch. 9. (c) Lounasmaa, M.; Tolvanen, A. In Monoterpenoid Indole Alkaloids; Saxton, J. E., Ed. In The Chemistry of Heterocyclic Compounds; Taylor, E. C., Ed.; J. Wiley: Chichester, 1994; Vol. 25, Supplement to Part 4, Ch. 3. (d) Szántay, C. Ibid. Ch. 9.

3. (a) Amat, M.; Hadida, S.; Pshenichnyi, G.; Bosch, J. J. Org. Chem. 1997, 62, 3158. (b) Amat, M.; Hadida, S.; Bosch, J. Tetrahedron Lett. 1993, 34, 5005.

4. For recent examples on the synthesis of 2-aryl- and 2-heteroarylindoles by $\mathrm{Pd}(0)$-catalyzed cross-coupling reactions from 2-indolylorganometals, see the following. Zinc derivatives: (a) Liu, S.-F.; Wu, Q.; Schmider, H. L.; Aziz, H.; Hu, N.-X.; Popovic, Z.; Wang, S. J. Am. Chem. Soc. 2000, 122, 3671. (b) Sakamoto, T.; Kondo, Y.; Takazawa, N.; Yamanaka, H. J. Chem. Soc., Perkin Trans. 1 1996, 1927. (c) Sakamoto, T.; Kondo, Y.; Takazawa, N.; Yamanaka, H. Heterocycles 1993, 36, 941. Boron derivatives: (d) Merlic, C. A.; You, Y.; McInnes, D. M.; Zechman, A. L.; Miller, M. M.; Deng, Q. Tetrahedron 2001, 57, 5199. (e) Koning, C. B.; Michael, J. P.; Rousseau, A. L. J. Chem. Soc., Perkin Trans. 1 2000, 1705. (f) Ishikura, M.; Agata, I.; Katagiri, N. J. Heterocyclic Chem. 1999, 36, 873. (g) Murase, M.; Watanabe, K.; Kurihara, T.; Tobinaga, S. Chem. Pharm. Bull. 1998, 46, 889. (h) Johnson, C. N.; Stemp, G.; Anand, N.; Stephen, S. C.; Gallagher, T. Synlett 1998, 1025. Tin derivatives: (i) Li, J. J.; Yue, W. S. Tetrahedron Lett. 1999, 40, 4507. (j) Wendeborn, S.; Berteina, S.; Brill, W. K.-D.; De Mesmaeker, A. Synlett 1998, 671. (k) Danieli, B.; Lesma, G.; Martinelli, M.; Passarella, D.; Peretto, I.; Silvani, A. Tetrahedron 1998, 54, 14081. (l) 
Gmeiner, P.; Kraxner, J.; Bollinger, B. Synthesis 1996, 1196. (m) Hudkins, R. L.; Diebold, J. L.; Marsh, F. D. J. Org. Chem. 1995, 60, 6218. (n) Labadie, S. S.; Teng, E. J. Org. Chem. 1994, 59, 4250. (o) Fukuyama, T.; Chen, X.; Peng, G. J. Am. Chem. Soc. 1994, 116, 3127. (p) Palmisano, G.; Santagostino, M. Helv. Chim. Acta 1993, 76, 2356.

5. Amat, M.; Hadida, S.; Sathyanarayana, S.; Bosch, J. Tetrahedron Lett. 1996, 37, 3071.

6. For the generation and $\mathrm{Pd}(0)$-catalyzed cross-coupling reactions of 4-, 5-, and 6-methoxy substituted 3-indolylzinc derivatives, see: Amat, M.; Seffar, F.; Llor, N.; Bosch, J. Synthesis 2001, 267.

7. (a) Batcho, A. D.; Leimgruber, W. Org. Synth. 1985, 63, 214. (b) Clark, R. D.; Repke, D. B. Heterocycles 1984, 22, 195.

8. (a) Illi, V. O. Synthesis 1979, 136. (b) Bergman, J.; Pelcman, B. Tetrahedron 1988, 44, 5215.

9. For reviews, see: (a) De Lucchi, O.; Miotti, U.; Modena, G. Org. React. 1991, 40, 157. (b) Grierson, D.; Husson, H.-P. In Comprehensive Organic Synthesis; Trost, B. M., Fleming, I. Eds.; Pergamon Press: Oxford, 1991; Vol. 6, pp 909-947. (c) Kennedy, M.; McKervey, M. A. Ibid., Vol. 7, pp 193-216.

10. Amat, M.; Bosch, J. J. Org. Chem. 1992, 57, 5792.

11. (a) Rubiralta, M.; Diez, A.; Bosch, J.; Solans, X. J. Org. Chem. 1989, 54, 5591. (b) Bennasar, M.-L.; Zulaica, E.; Ramirez, A.; Bosch, J. J. Org. Chem. 1996, 61, 1239.

12. (a) Amat, M.; Bennasar, M.-L.; Hadida, S.; Sufi, B. A.; Zulaica, E.; Bosch, J. Tetrahedron Lett. 1996, 37, 5217. (b) Bennasar, M.-L.; Jiménez, J.-M.; Vidal, B.; Sufi, B. A.; Bosch, J. J. Org. Chem. 1999, 64, 9605.

13. Allen, M. S.; Gaskell, A. J.; Joule, J. A. J. Chem. Soc. (C) 1971, 736.

14. Ishibashi, H.; Akamatsu, S.; Iriyama, H.; Hanaoka, K.; Tabata, T.; Ikeda, M. Chem. Pharm. Bull. 1994, 42, 271.

15. Sundberg, R. J.; Parton, R. L. J. Org. Chem. 1976, 41, 163.

16. Rubiralta, M.; Diez, A.; Reig, I.; Castells, J.; Bettiol, J.-L.; Grierson, D. S.; Husson, H.-P. Heterocycles 1990, 31, 173.

17. Methyl 2-chloropyridine-4-carboxylate was prepared in 97\% yield by treatment (reflux, $12 \mathrm{~h}$ ) of methyl isonicotinate $N$-oxide $(4.6 \mathrm{~g}, 30 \mathrm{mmol})$ with $\mathrm{POCl}_{3}(11 \mathrm{~mL})$ in $\mathrm{CHCl}_{3}$ (11 mL). 